\title{
Los retos políticos de la inmigración
}

\author{
ANA MARÍA LÓPEZ SALA \\ Universidad de La Laguna \\ «La globalización económica desnacionaliza la economía nacio- \\ nal, mientras que la inmigración renacionaliza la política.»
}

Saskia Sassen

Resumen. Desde la época de las grandes migraciones ultramarinas, hace algo más de un siglo, los estudiosos de las migraciones han elaborado marcos de interpretación de este fenómeno social.

La inmigración ha emergido recientemente en las sociedades occidentales como un tema de interés político. Así, la incorporación de los estudios migratorios desde la perspectiva de las disciplinas dedicadas al análisis del gobierno y la política es una de las novedades más interesantes en el reciente panorama teórico de las migraciones.

El objetivo de este artículo es detenerse en algunos de los más significativos efectos de la inmigración en la esfera política de las sociedades de acogida a partir de una premisa de partida: las migraciones internacionales han difuminado tres tipos de «fronteras» políticas profundamente interrelacionadas en las sociedades modernas. En primer lugar, los límites físicos, territoriales del Estado; en segundo lugar, el perímetro de la ciudadanía y de la pertenencia definida por la asignación de derechos de naturaleza liberal y, en tercer lugàr, el contorno de las comunidades nacionales en términos de identificación o afiliación cultural.

\begin{abstract}
Since the dawn of the age of mass migration, over a century ago, scholars have provided general explanations and analytical frameworks for the phenomenon of human migration.

Immigration has emerged as an important political issue in receiving countries during the ' $90 \mathrm{~s}$. In the disciplines and subdisciplines associated with the study of politics and government, including political sociology, political science, public policy and public administration and international relations, only recently has migration emerged as a field of study.

This article centres around the political focus in the analysis of international migration and treats the effects of international migration on political boundaries: territorial borders of states (and the question of control) and the impact of immigration on citizenship and the cultural boundaries of political community.
\end{abstract}




\section{EL «NUEVO» INTERÉS POLÍTICO DE LA INMIGRACIÓN}

Es ya un lugar común que la inmigración se ha transformado en este comienzo de siglo en uno de los motores fundamentales de transformación económica, social y política. Aunque puede afirmarse que no ha sido un tema particularmente ausente del discurso público y mediático durante las últimas décadas, es posible, sin embargo, sostener que el análisis de sus ramificaciones políticas no ha tenido un papel destacado en la evolución del «corpus teórico migratorio» elaborado por la academia desde finales del xix. De hecho, como he sostenido en otro texto ${ }^{1}$ en alusión a una primera y seminal dimensión política del fenómeno, los Estados han intervenido desde su misma creación en los flujos interfronterizos de personas, gestionando y controlando la entrada, salida y el establecimiento de población foránea en el interior de sus territorios. A pesar de ello, el papel del Estado como variable independiente, como actor cuyas iniciativas han tenido un impacto en los procesos migratorios ha sido subestimado hasta fechas recientes.

Son hoy mucho más intensos y variados los intentos, desde distintas disciplinas, como la Sociología, la Ciencia Política o las Relaciones Internacionales, por incluir al Estado en el análisis de los flujos migratorios ${ }^{2}$. Así, la incorporación de los estudios migratorios desde la perspectiva de las disciplinas dedicadas al análisis del gobierno y la política es una de las novedades más interesantes en el reciente panorama teórico de las migraciones.

No es casual el interés que este fenómeno suscita actualmente entre los teóricos políticos. Puede afirmarse que desde los años setenta hemos asistido a un paulatino proceso de politización de la inmigración o a la transformación de las migraciones en un objeto de disputa o controversia política. La influencia de la cuestión migratoria en el ámbito de lo político tiene una doble vertiente que se resumiría en la diferencia que establece el inglés entre los términos politics y policy y sus equivalentes política y políticas en castellano.

En primer lugar, los extranjeros se han transformado en objeto de la política a través de la elaboración de políticas de inmigración y extranjería. Estas medidas públicas, que concentran nuevos recursos estatales, abordan no sólo el control de los flujos migratorios, es decir, la entrada de inmigrantes en el territorio de los Estados de acogida, sino la integración, que da forma al acceso de estas comunidades a derechos de naturaleza social y económica, así como

\footnotetext{
${ }^{1}$ Véase A. M. López Sala, Inmigrantes y Estados: la respuesta política ante la cuestión migratoria, Barcelona, Anthropos, 2002 (en prensa).

${ }^{2}$ Véase J. Hollifield, «The Politics of International Migration. How can we bring the State Back in», en C. Brettel y J. Hollifield (eds.), Migration Theory: Talking across disciplines, Londres, Routledge, 2000, y A. Zolberg, «Matters of State: Theorizing Immigration Policy», en C. Hirschman, P. Kasinitz y J. De Wind, (eds.), The Handbook of International Migration. The American Experience, Londres, Russell-Sage, 1999.
} 
la regulación de la nacionalidad, que impacta sobre las posibilidades de incorporación de los inmigrantes a la membrecía política de los Estados receptores ${ }^{3}$.

La cuestión migratoria se ha convertido asimismo en un fenómeno con gran influencia en la realidad política de los países de acogida por el impacto que genera en las estructuras institucionales de nuestro sistema liberal democrático, como destaca Zapata en uno de los artículos pioneros sobre esta materia en nuestro país (R. Zapata, 2000) ${ }^{4}$. Pero la inmigración no sólo se ha convertido en un objeto de transformación, sino de creación institucional. Las políticas migratorias se configuran siguiendo una serie de ideas e intereses, la mayor de las veces en conflicto, y promueven la aparición de nuevos órganos, de nuevas agencias gubernamentales encargadas, por lo general, de su gestión interna. No es ésta cuestión baladí en el caso español. La inmigración ha sido, desde mediados de los años ochenta, materia de producción normativa y de creación de nuevas estructuras y órganos en la administración. Baste señalar la constitución en el año 2000 de una Delegación del Gobierno para la Extranjería y la Inmigración en el organigrama del Ministerio del Interior o la aparición de Consejerías y Concejalías de Inmigración en algunas comunidades autónomas y en diversos municipios españoles. La preparación de planes administrativos ha acompañado, asimismo, la creación de las «agencias de la integración» con el impulso inicial que recibieron a partir del Plan de 1994.

Este impacto en la esfera administrativa se ha extendido, igualmente, a las organizaciones humanitarias y sociales. El fenómeno migratorio ha sido incorporado como materia de intervención directa en las agendas de muchas ONGs, de las organizaciones confesionales, sindicales y de empresarios, de las federaciones de municipios y se ha integrado paulatinamente en los programas de estudios de universidades, centros de investigación e instituciones de formación continua.

La inmigración se ha transformado asimismo en un hecho social que ha terminado por afectar profundamente al núcleo sensible del poder político por su impacto en algunos de los conceptos articuladores del proceso de construcción nacional, como la ciudadanía, la identidad nacional o los derechos liberales. Citando de nuevo a Zapata, la presencia inmigrante, «tiene un efecto espejo, en tanto que nos obliga a plantearnos directamente nuestra tradición cultural liberal democrática. Prácticamente todas las categorías que han ayudado a describir y a explicar nuestro sistema liberal democrático son objeto de revi-

${ }^{3}$ Véase A. M. López Sala, «La gestión política de la migración», en F. Colom (ed.), $E l$ espejo, el crisol y el mosaico. Modelos políticos para el Multiculturalismo, Barcelona, Anthropos, 2001.

${ }^{4}$ En opinión de este autor catalán, el enfoque institucional de la ciencia política sostiene que el primer contacto que tiene el inmigrante con nuestra sociedad es con sus instituciones y que la forma en que está estructurada la esfera pública está directamente relacionada con un tipo de actitud, de conducta y de práctica que en numerosas ocasiones entra en tensión con la de los inmigrantes. Son ejemplos particularmente expresivos las fricciones que se producen en espacios como la escuela, las consultas médicas o los servicios sociales. 
sión» (R. Zapata, 2000, p. 15), aún más en un contexto caracterizado por el incremento de la presión globalizadora y la aparición de entidades supranacionales como la Unión Europea.

La reformulación política que parece acarrear la llegada y el establecimiento de población extranjera hunde sus raíces en la propia constitución de los Estados nacionales o, más en particular, en el artificio o construcción social de nuestros Estados como entidades internamente homogéneas en términos culturales y étnicos. Algunos autores han defendido que el reconocimiento de la diversidad cultural es contradictoria con el proceso histórico de formación de los Estados-nación en Europa y recuerdan que las naciones-estado modernas son, por definición, etnófagas o etnocidas, ya que la unificación política y el esfuerzo de homogeneidad cultural que llevaron aparejados entrañó la desaparición, o, al menos, la reinterpretación de los particularismos (D. Schnapper, 1992). La pulsión identitaria en las múltiples formas que han adoptado los «movimientos de minorías» ha servido no sólo para profundizar en el reconocimiento de la condición multicultural de nuestras sociedades nacionales modernas ${ }^{5}$, sino para llevar a la palestra política, entre otras cuestiones, las posibilidades de gestión interna de la pluralidad en un contexto de diversificación de ethos y formas de vida y el gobierno de las reclamaciones de reconocimiento de esta pluralidad en la esfera pública ${ }^{6}$. De esta situación se desprenden los debates en torno a los derechos de naturaleza colectiva, así como las posibles fórmulas políticas de incorporación a la sociedad mayoritaria de las nuevas minorías inmigrantes.

Pero las ramificaciones políticas de la inmigración no se detienen aquí. El fenómeno migratorio ha modificado considerablemente las relaciones entre Estados convirtiéndose en un elemento fundamental de la agenda europea y de los contactos bilaterales. Por añadidura, la inmigración ha creado nuevas clientelas y ha llegado a alterar el equilibrio de fuerzas en algunos países europeos donde los partidos con un discurso racista y contrario a la inmigración han ganado apoyo electoral. Es especialmente preocupante el apoyo que recientemente recibió Jean-Marie Le Pen en las dos vueltas de las presidenciales francesas y el éxito del Leefbaar Nederlând en las elecciones holandesas del pasado mes de mayo. En todos estos «logros políticos» indican los analistas, síntomas, en opinión de algunos de ellos, de una democracia enferma y origen de un estado de emergencia democrática ${ }^{7}$, la cuestión migratoria y la presencia de comunidades extranjeras aparece como telón de fondo, como causa última de nuevos apoyos electorales entre la población autóctona.

\footnotetext{
${ }^{5}$ Véase J. Rex, Ethnic Minorities in the Modern Nation State, Londres, MacMillan, 1996; T. Hammar, Democracy and the Nation State: Aliens. Denizens and Citizens in a World of International Migration, Avebury, Aldershot, 1990, y S. Castles y M. Miller, The Age of Migration. International Populations Movements in the Modern World, Londres, MacMillan, 1993.

${ }^{6}$ Véase C. Westin y J. Dacyl (eds.), Governance of Cultural Diversity: Selected Aspects, Center for Research in International Migration and Ethnic Relations, Suecia, Universidad de Estocolmo.

${ }^{7}$ P. Flores D'arcais, Estado de alerta en Europa, El País, 28 de abril de 2002.
} 
La diversidad etnocultural de las sociedades modernas se plantea, asimismo, como fuente de conflicto social y democrático. A los episódicos desórdenes callejeros protagonizados por jóvenes autóctonos e inmigrantes de segunda generación se unen ciertos ataques o asesinatos racistas, pero también la construcción social y opinática de ciertas comunidades como radicalmente inasimilables y presuntamente antidemocráticas, como se indica, especialmente, en el caso de las minorías musulmanas. Son muchas las voces que sostienen que la presencia de extranjeros y la formación de comunidades étnicas puede minar la identidad nacional, la unidad cultural de las sociedades receptoras, promover la división social y socavar seriamente la democracia y los sistemas del bienestar.

La emergencia de las relaciones interraciales y de la inmigración como cuestiones centrales de la agenda política europea se consolida a lo largo de la década de los noventa y es resultado, probablemente, de un doble efecto: de la «sensación de crisis» que provoca la inmigración (S. Sassen, 2001; C. Brettel y J. Hollifield, 2000) y de la transformación de las comunidades inmigrantes en actores políticos influyentes que ejercen demandas a las instituciones administrativas y democráticas de los países de acogida. Esta acción política de naturaleza colectiva es resultado no sólo del acceso de los inmigrantes al demos, a través de la atribución de la nacionalidad, sino de la progresiva concesión a los extranjeros de derechos de naturaleza económica, social y política tradicionalmente otorgados, en exclusiva, a los ciudadanos. La consecuencia más destacable ha sido, sin lugar a dudas, el efecto de fragmentación interna de los sujetos sometidos al gobierno del Estado a través de la aparición de nuevas categorías de residentes quasi-ciudadanos, que ha dado lugar a un nuevo léxico que incluye términos tan exitosos como margizens y denizens ${ }^{8}$.

Las demandas de las comunidades extranjeras han provocado rechazo o reacciones de apoyo y solidaridad. $Y$ es que resulta indiscutible que la inmigración crea fuertes controversias en la esfera pública y de opinión. Así se observa, por ejemplo, en el caso español, en donde tras una período de cierta indiferencia surge con fuerza como uno de los «problemas-clave», junto al desempleo y el terrorismo ${ }^{9}$. Es incuestionable el potencial efecto de esta corriente de opinión en el diseño de las políticas de inmigración y de integración, presididas por el objetivo del reclutamiento de comunidades «con vocación de integración» y la contención y limitación de las entradas. Ambas orien-

${ }^{8}$ Sobre el término «margizen» véase M. Martiniello, Migration, Ethnicity and Ethno-National Identities in the European Union, Aldershot, Avebury, 1995. Sobre el término «denizen» puede consultarse T. Hammar, Democracy and the Nation State. Aliens. Denizens and Citizens in a World of International Migration, Avebury, Aldershot, 1990.

- Véase L. Gimeno González, Actitudes ante la inmigración. Relación entre las investigaciones cualitativas y cuantitativas, Colección Opiniones y Actitudes, núm. 34. Madrid, CIS, 2001, y R. Simon y J. Lynch, «A Comparative Assessment of Public Opinion toward Immigrants and Immigration Policies», en International Migration Review, vol. 22, núm. 2, 1999. 
taciones, en opinión de una buena parte de la clase política, pueden impedir tanto el racismo social como el racismo de Estado.

Resulta imposible abordar en un artículo de estas características cada una de las dimensiones políticas mencionadas en párrafos anteriores. Mi propósito es detenerme en algunos de los retos políticos que plantea la inmigración, así como en los desarrollos teóricos y en los argumentos que han contribuido a construir un paradigma, o un enfoque específico de la cuestión migratoria desde esta «óptica política». Es conveniente subrayar, a modo de presentación, que aparece entre los especialistas un gran consenso en torno a la idea de que la migración internacional ha difuminado tres tipos de «fronteras» políticas profundamente interrelacionadas en las sociedades modernas. En primer lugar, los límites físicos, territoriales del Estado; en segundo lugar, el perímetro de la ciudadanía y de la pertenencia definida por la asignación de derechos de naturaleza liberal y, en tercer lugar, el contorno de las comunidades nacionales en términos de identificación o afiliación cultural. En opinión de Bauböck, «estas fronteras políticas constituyen rasgos esenciales de las sociedades modernas [...] A pesar de ello, la inmigración es un fenómeno que causa un considerable desconcierto, ya que afecta al significado social, la permeabilidad, la localización espacial y la estabilidad temporal de estos tres tipos de límites políticos» (R. Bauböck, 1998, p. 17). Como veremos a lo largo de las próximas páginas, la erosión que la inmigración ha causado en cada una de estas barreras políticas se ha producido como consecuencia de un cúmulo de desajustes entre distintas esferas tradicionalmente vinculadas en las democracias de corte liberal.

\section{MIGRACIONES, FRAGMENTACIÓN TERRITORIAL DEL SISTEMA DE ESTADOS Y SOBERANÍA}

\section{Fronteras territoriales del Estado y migraciones internacionales}

Las cuestiones planteadas hasta el momento aluden, en primera instancia, a la significación que adquieren en las migraciones internacionales el establecimiento de fronteras políticas territoriales. Aunque la inmigración, entendida como el desplazamiento espacial de personas, no es un fenómeno nuevo en los anales de la historia, se dota de un nuevo contenido con la modernización, el advenimiento de los Estados nacionales y la transformación de la sociedad internacional en una red de Estados soberanos rivales.

Como ha explicado Ernest Gellner en su clasificación de tipos de sociedades humanas, la noción de sociedades compuestas por individuos vinculados al territorio de un Estado carece de significación en condiciones de escasa movilidad. En las sociedades agrarias, las fronteras estatales no tenían un efecto significativo sobre las fronteras sociales porque el desplazamiento de población no se hacía efectivo. La movilidad que acarrea la industrialización da nueva forma a las estructuras sociales rompiendo la unidad territorial e institucional 
entre el hogar y el lugar de trabajo y dotando de nueva significación a las fronteras geográficas, políticas y culturales de los Estados (E. Gellner, 1988).

La era de la industrialización y del nacionalismo crea un doble proceso de rigidez o de territorialidad excluyente a escala doméstica e internacional. Internamente, la emergencia de una administración pública moderna con una burocracia profesional promueve un tipo de estructura jerárquica de gobierno territorial que requiere la consiguiente estructura administrativa. El desarrollo de una ciudadanía democrática enriquecida con derechos de naturaleza social y económica vinculada a los sistemas del bienestar ha densificado la importancia $\mathrm{y}$ rigidez de la estructura territorial interna y externa de los Estados modernos ${ }^{10}$.

Sin embargo, lo que dota a la cuestión migratoria de su carácter eminentemente político es que el cruce de las fronteras de un determinado Estado, implica, por definición, al menos a medio plazo, un cambio en la jurisdicción y la membrecía, desactivándose la clásica identificación entre ciudadanía y territorio.

Probablemente sea Aristide Zolberg el primer autor que insiste en abordar los flujos migratorios desde una perspectiva política. La mención a su pensamiento resulta de vital importancia en el enfoque que estamos tratando. El interés de sus aportaciones reside en dos supuestos desarrollados en su trabajo desde principios de los años ochenta. En primer lugar, Zolberg subraya que la negación del papel del Estado y de sus políticas concretas constituye uno de los principales defectos o debilidades de las teorías sobre las migraciones (A. Zolberg, 1981 y 1989). Esta crítica se remonta a los primeros enunciados de la teoría migratoria desde la aportación pionera de Ravenstein a finales del XIX en sus leyes de la migración ${ }^{11}$. En su opinión, esta omisión podría ser resultado de que los procesos migratorios son fenómenos que se desvían de la norma en una visión consensuada de la sociedad internacional como una entidad constituida por Estados reificados y autónomos, sustentados en el dominio sobre un territorio, que autorreproducen sus sistemas sociales y culturales y cuya población se renueva endógenamente (A. Zolberg, 1981). La condición «territorial» de los Estados modernos o para decirlo con más precisión, la condición del Estado como una unidad territorial estable, y la consecuente segmentación del sistema internacional explica, en buena medida, por qué la inmigración se convierte en un fenómeno tan irritante (R. Bauböck, 1998). Este axioma elude tanto la noción de diáspora o de comunidad transnacional, como el nutriente demográfico y social que los flujos incorporan

10 De hecho, uno de los temores más reiterados por la opinión pública y las administraciones es la presión de la población inmigrante sobre recursos escasos. El impacto de la inmigración en los sistemas del bienestar ha sido un objeto de estudio prioritario entre los teóricos apuntándose, por ejemplo, su efecto en «la politización» del Estado del bienestar o de los derechos sociales (veáse T. Faist, 1996). En el marco de estas preocupaciones se encuentra la idea de que las contribuciones que realizan al Estado de acogida son menores que los recursos que demandan, de ahí el éxito de la expresión «el negativo balance fiscal de la inmigración» (J. Arango, 1994).

${ }^{11}$ Veáse A. Zolberg (1999) y A. M. López Sala (2002). 
a las sociedades de acogida. $Y$ es que la inmigración debe concebirse en cualquier tipo de análisis como un fenómeno genuinamente transnacional no sólo en relación al cruce de fronteras nacionales, sino como consecuencia de la variedad de afiliaciones sociales y políticas que crea. Las migraciones internacionales transnacionalizan no sólo las sociedades de origen y de acogida, sino las políticas, los derechos y los términos de la pertenencia más allá de la rigidez de las fronteras territoriales ${ }^{12}$.

En definitiva, lo que concede el carácter político a las migraciones internacionales, es, en opinión de Zolberg, en primer lugar, su condición de fenómeno transnacional, de «fenómeno que atraviesa fronteras», lo que supone, por tanto, su sometimiento a las condiciones establecidas desde el Estado y el derecho internacional al movimiento de personas tanto desde los lugares de origen, como a los lugares de acogida, sean éstos, o no, espacios de asentamiento definitivo. Los flujos migratorios se ven, en consecuencia, afectados por la acción política, por la normativa de los Estados de salida y de acogida, así como por la multitud de disposiciones sobre esta materia en convenios multinacionales y binacionales ${ }^{13}$.

El asentamiento incorpora además una nueva área de influencia, la de las disposiciones sobre integración y política social de los gobiernos de acogida. Se multiplica, se densifica de esta manera, la jurisdicción migratoria, el perímetro del poder, sobre los flujos de personas.

\section{Migración, soberanía y control fronterizo}

No debemos olvidar que la política migratoria es un campo de intervención pública sometida a múltiples tensiones. Mientras que la posibilidad de abandonar el Estado del que se es ciudadano es un derecho inalienable que afecta a la libertad del individuo, no existe el derecho equivalente a ser aceptado en otra comunidad política. La Declaración Universal de los Derechos Humanos es taxativa en este punto. El artículo 13 reconoce la libertad de movimiento y residencia en el interior de las fronteras de un Estado, así como el derecho de toda persona a abandonar cualquier país (incluido el suyo) y a retornar al país del que se es ciudadano. No existe, sin embargo, en esta declaración, ningún artículo que reconozca el derecho concomitante a entrar o residir en cualquier país del que no se es ciudadano. Existe, de hecho, en la comunidad internacional, un consenso universal y poco ambiguo en torno a la legitimidad de los Estados para decidir qué extranjeros desean recibir. El derecho de entrada

${ }^{12}$ Véase R. Bauböck, A. Heller y A. Zolberg (1996), Y. Soysal (1994), D. Jacobson (1996) y más recientemente S. Castles y A. Davidson (2000) y R. Koopmans y Statham (2000)

${ }^{13}$ Las aportaciones de Zolberg son posteriormente incorporadas de forma sistemática a los estudios migratorios por, entre otros, el politólogo sueco Tomas Hammar, uno de los teóricos pioneros en el análisis comparado de políticas de inmigración y en el fenómeno migratorio como un proceso secuencial de cruce de fronteras de naturaleza política (T. Hammar, 1985, 1990). 
se encuentra, por tanto, supeditado al principio de autodeterminación colectiva de una comunidad nacional y asî se reconoce - es un principio no disputadoen el derecho internacional.

Y es que, pese a los procesos de globalización y de creación de instituciones políticas de carácter supranacional, la competencia de los Estados para controlar las entradas y el establecimiento de inmigrantes constituye una de las últimas prerrogativas de su soberanía, entendida ésta, en términos sociológicos, como un mecanismo de exclusión o cierre a través del cual las sociedades regulan su composición interna. Como indicaba Hannah Arendt, la aplicación del principio de soberanía en ningún ámbito es más absoluta que en materia de migración, naturalización, nacionalidad y expulsión.

Adicionalmente, las migraciones internacionales permiten expresar la tensión entre los intereses de los individuos y los intereses de las sociedades organizadas en Estados. Desde el punto de vista de los primeros, el mundo se presenta como un campo para la optimización de las oportunidades vitales mediante la opción entre diferentes alternativas (entre ellas, la de asentarse o trabajar o llegar a ser ciudadano de un país que no es el de origen). De otro lado, el sistema político internacional se presenta como un conjunto de Estados potencialmente rivales que intentan maximizar sus intereses colectivos actuando, entre otras esferas, sobre las entradas y las salidas de personas, sobre el acceso al mercado de trabajo y sobre el ingreso en la comunidad política. Esto nos remite, por tanto, a algunas de las discusiones más recientes que han puesto en entredicho la asunción esencial del liberalismo que prima los intereses de los individuos frente a los de las comunidades. Las distintas modulaciones de la teoría liberal han repercutido así sobre la legitimación del derecho de emigración.

Las reflexiones en torno a este derecho aluden, en última instancia, a la existencia de un litigio entre el concepto de libertad y el de comunidad. Desde la perspectiva del libertarismo, representada por autores como Nozick, las relaciones económicas entre nacionales y extranjeros no deberían ser violadas por constricciones externas a las que conllevan dichas transacciones. Las políticas de inmigración, por tanto, no deberían frenar los flujos de entrada. Las fuerzas del mercado se encargarán de determinar las posibilidades de establecimiento e instalación de inmigrantes (véase W. Kymlicka, 1995). Una posición liberal más clásica, como la expresada por Rawls en su Teoría de la Justicia, concluye que hay muy pocas restricciones a la inmigración que puedan ser moralmente defeñdibles (véase J. Carens, 1995). Una versión más del liberalismo, como la de Walzer, ha subrayado el derecho de las comunidades a decidir a quienes admiten con el fin de preservar su identidad colectiva y los bienes individuales que se derivan de ella. Su posición permite, por tanto, ciertas restricciones a la entrada de extranjeros (M. Walzer, 1983). Dos rasgos controvertidos de su teoría son la noción de que la soberanía política constituya un valor absoluto (una postura cada vez más desafiada por la evolución de la normativa sobre 
los derechos humanos universales) y la visión de que las únicas comunidades con carácter sean las que presentan rasgos etnoculturales comunes. Sin embargo, no debemos olvidar que las razones morales a las que se alude en estas posiciones no impregnan, por lo general, la práctica política. Las decisiones de los gobiernos en materia de inmigración se ven sometidas a la necesidad de conciliar conflictos entre actores o instituciones con diversos valores e intereses. Por ello, las políticas de inmigración parten de la prerrogativa del Estado para determinar su política interna y externa, y en ellas juegan un papel significativo los costes políticos de sus intervenciones y la competencia sobre recursos escasos. Las consideraciones morales incorporadas en el derecho internacional y en las constituciones nacionales se han reflejado fundamentalmente y, a veces, ni tan siquiera, en la política de refugio y en las disposiciones legales que regulan la reagrupación familiar y los derechos de menores y apátridas $(\mathrm{H}$. Adelman, 1995).

Las políticas de control y regulación de flujos han sido, además, un terreno propicio para la controversia porque en ellas confluye la tensión entre el principio de soberanía nacional y el de la salvaguardia de los derechos humanos. Los Estados reúnen gran parte de las competencias a la hora de decidir qué extranjeros desean admitir, pero las medidas adoptadas en esta materia han entrado en contradicción, en ciertos casos, con el respeto por los derechos fundamentales, algo que alude inevitablemente a los límites del control ${ }^{14}$. Esto es evidente en muchos de los procedimientos utilizados en materia de asilo o en la ejecución de las medidas sancionadoras.

En conclusión, la cuestión migratoria y, en especial, su dimensión como fenómeno de cruce fronterizo, ha afectado al concepto de soberanía por el desarrollo de normas de carácter supranacional que limitan la autonomía del Estado y que producen, en cierto sentido, una descentralización de la misma.

La persistencia de flujos de inmigración irregular a pesar de las férreas medidas de control fronterizo tiene también un impacto destacado sobre los gobiernos, en especial sobre la legitimación de sus políticas. Aunque en la mayor parte de los países existe cierto acuerdo entre las fuerzas políticas y sociales sobre la necesidad de controlar eficazmente los flujos de entrada, este acuerdo no es unánime en cuanto a la forma de aplicación de las medidas de control de los flujos, la restricción de las entradas de familiares y el endurecimiento de las políticas de refugio. Algunas acciones de control interno (como las expulsiones o las detenciones policiales) han sido profundamente impopulares. Por añadidura, las decisiones en materia de control de flujos - son tomadas por la administración y no suelen ser objeto de consulta. La opinión pública, las asociaciones de defensa de los derechos humanos y la prensa se han mostrado, sin embargo, como poderosos actores-escenarios de control de las actuaciones estatales en esta materia. Una de las nuevas fórmulas

${ }^{14}$ Véase H. van Amersfoort, «Migration: the Limits of Government Control», en New Community, vol. 22, núm. 2, 1996, pp. 243-257. 
de control migratorio desarrolladas por algunos Estados durante los años noventa, junto con las medidas clásicas de contención en frontera y control interno, ha sido la limitación efectiva de los derechos disfrutados por los inmigrantes en las sociedades de acogida, como factor de contención de los flujos en origen. Esta posición justificó la aprobación en 1994 de la proposición 187 en el Estado de California y protagonizó los debates políticos sobre la segunda Ley Pascua y la Ley Debré en Francia. El peligro de este tipo de medidas, como ha apuntado James Hollifield en su análisis sobre el caso francés y norteamericano, es que cuando «el Estado cruza la línea invisible y legítima sobre la regulación de la inmigración para proponer y ejecutar medidas que supongan un serio peligro para la sociedad civil y que entran en contradicción con los principios democráticos republicanos, surten efecto los controles sociales, institucionales, judiciales e ideológicos» (J. Hollifield, 1998, p. 24).

El segundo elemento que afecta a esta legitimación política está vinculado con la porosidad de las fronteras. La hipótesis del desajuste, enunciada recientemente por Cornelius, Martin y Hollifield, de acuerdo con la cual la distancia entre los objetivos y los resultados de las políticas de control es amplia y creciente, refleja fielmente este marco de preocupaciones. La dificultad de los Estados para controlar las fronteras de sus territorios explicaría la consolidación de un clima de opinión hostil a la inmigración, el incremento de la presión sobre los gobiernos para que se adopten políticas aún más restrictivas en esta materia y el aumento de la cooperación bilateral con el fin de mejorar los niveles de eficacia. La continuidad de los flujos de irregulares frente al desarrollo de los más sofisticados mecanismos de blindaje fronterizo es considerada, asimismo, fuente de descontento político, de frustración y de desconfianza en las instituciones gubernamentales (G. Freeman, 1997).

Hollifield señala que la confluencia de la lógica del mercado y la lógica de los derechos explica muchas de las dificultades actuales en el control de la inmigración en Europa y Estados Unidos. Esta nueva dinámica políticoeconómica parece haber roto o debilitado el vínculo tradicional entre ciclo económico y políticas liberales o restrictivas de inmigración (J. Hollifield, 1992) ${ }^{15}$. Estas explicaciones estructurales contrastan con otras hipótesis que sostienen que el fracaso se debe a la existencia, en el interior de los Estados, de poderosos grupos de interés que propician el mantenimiento de los flujos por razones económicas o a que los Estados no toman en consideración ciertos factores clave en el proceso migratorio. Uno de los artículos más elocuentes sobre esta cuestión es el del geógrafo holandés Hans van Amersfoort, quien introduce el concepto de «estructuras intermedias» para explicar los resultados poco óptimos de este tipo de políticas. Utilizando como ejemplo las medidas adoptadas en Holanda para frenar la entrada de población turca y marroquí

${ }^{15}$ Para una revisión crítica de las distintas aproximaciones teóricas al control de los flujos migratorios véase G. Sciortino, «Towards a Political Sociology of Entry Policies: conceptual problems and theoretical proposals", en Journal of Ethnic and Racial Studies, vol. 26, núm. 2, 2000. 
sugiere que las causas por las que este control ha resultado poco efectivo son: a) el desajuste entre la creación de políticas - que reflejan necesidades a corto plazo- y los efectos a largo plazo de los flujos migratorios, y b) la multiplicidad de intereses en el seno de la sociedad y del Estado $(\mathbf{H}$. van Amersfoort, 1996). La multiplicidad de intereses impide la formulación de instrumentos de regulación a largo plazo e interfiere en la implantación efectiva de las medidas adoptadas. La ejecución de las políticas va a estar, asimismo, seriamente influida por acciones concretas de los funcionarios implicados: policías, jueces, profesionales de la enseñanza, inspectores de trabajo, etc.

\section{EL IMPACTO DE LA INMIGRACIÓN EN EL PERÍMETRO DE LA CIUDADANÍA}

\section{La rigidez de las fronteras territoriales frente a la movilidad territorial de las comunidades y de los derechos}

Los movimientos migratorios parecen haber minado los modelos de diferenciación basados en la pertenencia a un Estado. Cuando los Estados expresan ese tipo de territorialidad excluyente frente a la movilidad de la población y de las comunidades se pone en evidencia la tensión entre varias esferas tradicionalmente imbricadas: a) la identificación entre ciudadanía y territorio, b) ciudadanía y derechos y c) ciudadanía e identidad cultural.

La formación histórica de los Estados nacionales ha venido acompañada de una definición de los individuos que se consideran miembros de la comunidad política y, en consecuencia, de la codificación de los derechos y obligaciones de todos los clasificados como tales. El imperativo de la organización de las poblaciones en un sistema de membrecía adquiere vigor con el tránsito a la modernidad. En este proceso, el tipo de control social ejercido por los Estados sobre sus poblaciones transforma en indispensable el desarrollo de mecanismos de identificación de la comunidad nacional. La pertenencia formal se organiza a través del estatus legal de ciudadano. Ëste recoge el conjunto de derechos y deberes de los miembros que forman parte de la comunidad política y es expresión de su alianza individual con el Estado. Las concepciones de la ciudadanía moderna se basan, en definitiva, en la organización de la población dentro de los límites territoriales de los Estados nación y en la pertenencia nacional como fuente de derechos y de deberes de los individuos. Esta conceptualización se ve alterada por la presencia en las sociedades de acogida de no ciudadanos - los extranjeros asentados- que disfrutan de derechos intrínsecos a tal condición ${ }^{16}$.

${ }^{16}$ Estos cambios han conducido también al desarrollo de distintos debates sobre la significación del transnacionalismo y de las comunidades transnacionales como fuentes de un nuevo tipo de membrecía. Las comunidades transnacionales son grupos cuya identidad no está sujeta, en primera 
En el estudio de los vínculos entre ciudadanía e inmigración es necesario comenzar estableciendo una distinción entre el concepto de ciudadanía formal y el de ciudadanía sustantiva. Formalmente, la ciudadanía está asociada a la condición de miembro de una comunidad política, de un Estado y, en consecuencia a tener una determinada nacionalidad. La completa pertenencia a la comunidad política está simbolizada por el derecho al sufragio y a la elección. La ciudadanía sustantiva se refiere al conjunto de derechos y deberes ligados con la titularidad de la condición de ciudadano de un determinado Estado (S. García, 1994).

Pero desde hace unas décadas se aprecia, como hemos apuntado, la presencia de grupos en el seno de las sociedades multiculturales que poseen fuertes vínculos con los Estados donde residen sin pertenecer a la comunidad política, además de disfrutar de una gama de derechos muy similar a la de los ciudadanos, a pesar de no ostentar tal condición. Por eso podemos sostener que la inmigración no sólo ha hecho desaparecer la tradicional identificación entre ciudadanía formal y sustantiva, sino que el progresivo asentamiento de comunidades extranjeras ha servido para poner en cuestión los rasgos que definían hasta el momento la condición ciudadana.

El estatus de pertenencia de los inmigrantes ha sido definido por Brubaker como un estatus políticamente anómalo. Esta afirmación se basa en su construcción teórica sobre la ciudadanía moderna como una categoría igualitaria, nacional, democrática, única y socialmente consecuente. «Este esquema se corresponde en ciertos aspectos con la distinción de Marshall entre los componentes civiles, políticos y sociales de la ciudadanía. La norma de una ciudadanía igualitaria se corresponde con el elemento civil, la ciudadanía democrática con el elemento político y la característica socialmente consecuente con el elemento social de su esquema. Este solapamiento sustantivo es sólo parcial. En el esquema de Marshall nada se corresponde con los caracteres nacionales y únicos de la pertenencia» (R. Brubaker, 1990, p. 399).

La ciudadanía es una categoría que en su acepción tradicional descarta gradaciones internas sobre el principio de igualdad entre todos aquellos que la disfrutan y que son portadores de los derechos y deberes que ésta asigna. Sin embargo, en las sociedades receptoras actuales apreciamos una proliferación de estatus de pertenencia parcial basada en el reconocimiento a los extranjeros de una gran parte de los derechos reservados a los ciudadanos. Esto se debe a que la pertenencia es una categoría más inclusiva que la ciudadanía formal.

La proliferación de estatus de pertenencia múltiple se opone al principio de pertenencia única. El reconocimiento de derechos a los extranjeros en las

instancia, a la identificación con un determinado territorio, algo que, indiscutiblemente, plantea un reto significativo para el modelo tradicional de pertenencia. Stephen Castles ha legado a apuntar recientemente que las afiliaciones y la «conciencia» transnacional pueden llegar a convertirse en un futuro en una de las formas mayoritarias de pertenencia entre los inmigrantes (S. Castles, 2002). 
sociedades de acogida es, en realidad, una expresión política de la pertenencia de los inmigrantes a diversas sociedades y Estado. A esta pertenencia múltiple en términos de asignación de derechos hay que añadir que la mayor parte de los extranjeros instalados en los países de acogida expresan una identidad híbrida. Esta identidad múltiple se ha reflejado jurídicamente en el aumento de los casos de doble nacionalidad.

Las nuevas modulaciones de la ciudadanía que se construyen como consecuencia de esa combinación de estatus parciales y múltiples entre los inmigrantes instalados cobra vigor en la perspectiva teórica desarrollada en torno al argumento de que el arraigo de la condición ciudadana a una membrecía singular y única a un Estado-nación no es hoy adecuado, ya que tal modelo está siendo severamente erosionado ${ }^{17}$. Esto significa la aparición de un nuevo tipo de Estado no construido exclusivamente alrededor del nexo entre membrecía y territorialidad, así como el reconocimiento de la aparición de nuevos tipos de pertenencia dual o múltiple (S. Castles y A. Davidson, 2000, y R. Bauböck, 1994a y 1998).

El desacoplamiento entre ciudadanía y territorio y ciudadanía y derechos ha impulsado la creación de, al menos, dos términos de cierto éxito en los estudios recientes sobre migraciones. Nos referimos al concepto de ciudadanía transnacional acuñado por Bauböck y al de ciudadanía postnacional enunciado por Soysal.

El interés de la aportación de Bauböck, en el marco de la preocupación por el impacto de las migraciones en la ciudadanía democrática, es que sugiere la aparición de un tipo de ciudadanía transnacional que reconoce en los inmigrantes el solapamiento de distintas afiliaciones políticas, lo que inevitablemente aumenta los vínculos entre los Estados de origen y de acogida (R. Bauböck, 1994a y 1994b, 2002). La tesis central de Soysal es que la experiencia inmigrante muestra que la ciudadanía nacional ha sido sustituida gradualmente por un modelo más universal de membrecía menos basado en consideraciones territoriales que en la noción de derechos del individuo. Soysal identifica el origen de este nuevo paradigma en el discurso transnacional de los derechos humanos. De acuerdo con su modelo, los derechos humanos universales sustituyen a los derechos nacionales y el individuo trasciende al ciudadano (Y. Soysal, 1994 y 1996) ${ }^{18}$.

La exclusión de los inmigrantes residentes de los mecanismos tradicionales de representación política se encuentra en contradicción, por añadidura, con el principio de que la pertenencia debe ser democrática. Esta posición supone

\footnotetext{
${ }^{7}$ Este tipo de propuesta también ha sido objeto de críticas en otros trabajos. Véase C. Joppke, Immigration and the Nation State: The United States, Germany and Great Britain, Oxford, Oxford University Press, 1999.

${ }^{18}$ David Jacobson señala, asimismo, la aparición de un nuevo régimen postnacional ligado a la ley internacional y al reconocimiento de los derechos humanos universales en donde los inmigrantes adquieren un estatus legal que eclipsa o supera la condición ciudadana anclada en la lógica del Estado nación (D. Jacobson, 1996).
} 
una seria limitación para la democracia representativa. De ahí los argumentos que defienden que el Estado receptor debe crear cauces para que los extranjeros lleguen a ser miembros de la comunidad política y fomentar otros mecanismos de participación cuando todavía no han adquirido la nacionalidad.

Tomas Hammar ha profundizado sobre estas cuestiones en su libro Democracy and the Nation State. Este politólogo sueco parte en su análisis de dos principios políticos básicos. El primero es la idea democrática de un gobierno representativo basado en las elecciones y en el sufragio universal. El segundo es el principio de Estado-nación, principio que afirma que el mundo está dividido en Estados soberanos y sólo aquellos que son ciudadanos pueden reclamar el derecho a participar en la vida política. Pero la presencia de una nutrida comunidad extranjera residente va a suponer un reto para ambos. «Si sólo se permite a los ciudadanos participar en la vida política se está produciendo en la práctica una significativa limitación de la democracia representativa. Una proporción sustancial de los adultos trabajadores no tienen representación a escala local, regional y nacional» (T. Hammar, 1990, p. 21). La concesión de derechos políticos a los extranjeros, sin embargo, cuestiona y entra en contradicción con el segundo principio mencionado». Hammar sostiene, al hilo sobre la limitación de la concesión de derechos políticos a los inmigrantes, que existe una razón muy importante para ello en la lógica del Estado contenida, precisamente, en la idea de democracia representativa: en sus propias palabras «como el demos es representado en los órganos de toma de decisiones, el derecho al sufragio activo y pasivo debe ser reservado a los ciudadanos que colectivamente constituyen the people. Las decisiones políticas deben reflejar sus decisiones e intereses, y no las de aquellos que no son ciudadanos» (T. Hammar, 1990, p. 129).

Similares argumentos encontramos en el reciente trabajo de Ruth Rubio, cuyo título sugiere, en parte, la posición de la autora: Immigration as a Democratic Challenge. Rubio parte de la necesidad de superar la falta de legitimidad política que acompaña el desacoplamiento entre la sociedad civil y política en contextos de inmigración. Su argumento es que aquellos que residen establemente en un Estado democrático deben ser considerados miembros de la comunidad política, por lo que deberían garantizárseles el conjunto de derechos y libertades que acompañan a su condición de sujetos políticos. Una concepción satisfactoria de la democracia liberal debe, en definitiva, construir un demos que incluya a todos aquellos individuos sujetos durante un significativo período de tiempo a la autoridad política del Estado, más allá de consideraciones sobre su afiliación a otras unidades políticas o a determinados rasgos culturales ( $R$. Rubio, 2000).

Sin embargo, no puede afirmarse, pese a los anteriores argumentos, que los inmigrantes carezcan realmente de voz política en las sociedades de destino. Uno de los hechos más novedosos en esta materia a los que hemos asistido desde los años setenta ha sido la concesión parcial de derechos políticos a 
los extranjeros residentes en algunos países de acogida. Los acuerdos para la concesión del derecho al voto en las elecciones locales siguiendo criterios de reciprocidad tienen cierta tradición en las legislaciones nacionales, una medida que en el caso de la UE se extiende a todos los ciudadanos de los países miembros. Pero desde mediados de los años setenta algunos países de Europa han concedido el voto en las elecciones locales y regionales a los extranjeros residentes, al margen de este criterio de reciprocidad. El país pionero en adoptar esta medida fue Suecia, que desde 1975 garantiza el derecho al voto a los extranjeros que lleven residiendo más de tres años. El ejemplo sueco ha sido seguido posteriormente por Holanda, Noruega y Dinamarca.

El hecho de que los inmigrantes carezcan del derecho al sufragio no ha impedido que en ciertas situaciones desempeñen un activo papel político. Ésta es precisamente la hipótesis que manejan algunos autores cuando sostienen que, a pesar de carecer del derecho al voto, las comunidades extranjeras establecidas en los países de acogida no pueden ser consideradas actores políticos insignificantes ${ }^{19}$. Tres son las razones que, por ejemplo, Catherine Whitol de Wenden menciona para argumentar esta posición: a) la existencia de formas de participación política no directamente ligadas al ejercicio electoral (la movilización sindical o el ejercicio del derecho de reunión y de manifestación), b) la creación, por parte del Estado de acogida, de órganos consultivos en materia de inmigración donde participan activamente las asociaciones étnicas (por ejemplo, los consejos interétnicos locales) y c) la presión política ejercida por las organizaciones de inmigrantes, cuyas reivindicaciones se han canalizado, en ciertos casos, a través de asociaciones autóctonas que se transforman en sus interlocutores frente a la administración (C. Whitol de Wenden y A. Hargreaves, 1993).

\section{Las barreras culturales de la ciudadanía}

Pero la construcción de los Estados modernos no sólo conllevó el establecimiento de estructuras políticas capaces de colmar el vacío creado por la desaparición de la sociedad feudal, sino que supuso indirectamente la unificación cultural del demos. Los diferentes intentos de definir la nación han incluido también una serie de elementos que debían compartir sus miembros: una historia y ascendencia común, una lengua y cultura y una socialización compartida a través de instituciones tales como la escuela ${ }^{20}$. En definitiva, la construcción de los Estados nacionales ha pasado por la centralización y " unificación de la identidad colectiva de los ciudadanos, lo que ha provocado,

\footnotetext{
${ }^{10}$ Sobre las diversas formas de participación política de los inmigrantes véase también $\mathrm{S}$. Vertovec, «Minority Associations, Networks and Public Policies: re-assessing relationships», en Journal of Ethnic and Racial Studies, vol. 25, núm. 1, 1999, pp. 21-42.

${ }^{20}$ Véase F. Ramírez y J. Boli, "The Political Construction of Mass Schooling: European origins and worldwide institutionalization», en Sociology of Education, vol. 60, 1987, pp. 2-17.
} 
asimismo, que la cultura fuera un elemento ausente de las teorías liberales más influyentes en torno a la ciudadanía.

No debemos olvidar que desde los principios liberales, la esfera política se caracteriza por el universalismo, mientras que las manifestaciones concretas de la identificación cultural se restringen a la esfera privada. Esto requiere de una estricta separación entre los derechos y las obligaciones políticas del individuo y su pertenencia a una serie de grupos conformados en torno a afiliaciones étnicas o religiosas. En la práctica, históricamente, la admisión a la ciudadanía implicaba la incorporación a la comunidad dominante a través de la asimilación cultural.

Sin embargo, la transformación en ciudadanos de un buen número de inmigrantes con una herencia cultural, étnica y religiosa muy diferente a la de la población autóctona ha alterado profundamente esta pretendida y tradicional homogeneidad cultural de la comunidad nacional, un fenómeno alimentado, asimismo, por el llamado revival étnico y las reclamaciones de las minorías indígenas y subnacionales.

Con independencia de los requisitos que los Estados establecen normativamente para la atribución o la adquisición de la nacionalidad, la inmigración reta la estructura clásica de las barreras culturales de la comunidad política a través de la incorporación de diversidad a las sociedades de acogida y la erosión de las barreras culturales tradicionales con procesos como la asimilación o la hibridación cultural e identitaria.

El debate en torno al reconocimiento de las colectividades culturalmente distintivas en el seno del Estado nacional ha suscitado una profunda polémica entre los teóricos. La preocupación de fondo es la de si el liberalismo, tal y como ha sido entendido durante los dos últimos siglos, puede hacer frente a estas reclamaciones, sobre todo porque tienen una dimensión colectiva tradicionalmente olvidada en los principios teóricos de la democracia liberal.

Las propuestas teóricas pergeñadas por los defensores del pluralismo cultural han adoptado varias formas. Encontramos, por un lado, posiciones que han defendido la necesidad de que el concepto de ciudadanía tenga en cuenta estas diferencias, de ahí los argumentos que apoyan la creación de una ciudadanía diferenciada, basada en el establecimiento de «derechos a la carta» en la que se incluyan los derechos culturales de grupo (I. Young, 1989) ${ }^{21}$.

Otras fórmulas, que promueven una gestión armónica de las diferencias en la esfera de la conformación de la comunidad nacional, se encuentran más

\footnotetext{
${ }^{22}$ «El sentido universal de la ciudadanía se encuentra en tensión con dos significados de la universalidad empotrados en las ideas políticas modernas: la universalidad como generalidad y la universalidad como igualdad de tratamiento. De esta forma la tradición liberal de la ciudadanía se basa en que la gente es igual y homogénea en su papel como ciudadanos y, por ello, deberían ser tratados como iguales por parte del Estado. En la práctica esto tiene dos problemas: excluye a aquellos grupos considerados incapaces de adoptar el punto de vista general y, en segundo lugar, la igualdad de tratamiento ayuda a mantener el sistema actual de grupos privilegiados que concentran el poder» (I. Young, 1989, pp. 250-251).
} 
o menos próximas a lo que se conoce como ciudadanía multicultural ${ }^{22}$, un término acuñado por Kymlicka al hilo de su análisis sobre la capacidad de los Estados para hacer frente a las reivindicaciones de los grupos culturalmente diferenciados (W. Kymlicka, 1995).

En opinión de Kymlicka, las comunidades inmigrantes no pretenden formas específicas de autogobierno, como las que reclaman las minorías nacionales, sino una serie de transformaciones en las instituciones y en la legislación de los países de acogida que les permitan participar en la sociedad en la que viven en términos de igualdad. Reclaman, en su opinión, un tipo de derechos - a los que denomina derechos poliétnicos- que no pueden ser, en sentido estricto, sustrato de una ciudadanía diferenciada. El reconocimiento de la especificidad cultural, defiende, es compatible con los principios liberales, y puede ser llevado a cabo en la práctica a través de lo que se han denominado, en el caso de los inmigrantes, políticas de integración multiculturales. De esta manera un Estado liberal puede aceptar y promocionar que un determinado grupo disfrute de ciertos derechos en contra de la sociedad mayoritaria - como en las políticas de discriminación positiva - derechos que les facilitarán no sólo expresar libremente su identidad en el interior de la sociedad mayoritaria, sino mantener su diferenciación cultural a lo largo de generaciones, así como hacer realidad su igualdad como ciudadanos al margen de diferencias culturales o lingüísticas.

Podríamos indicar, por tanto, a modo de conclusión ${ }^{23}$, que los procesos migratorios y el establecimiento de comunidades de origen extranjero han producido un apreciable desacoplamiento entre el demos y el ethnos. Queda hoy todavía sin resolver las posibles acciones que desde el Estado o la sociedad civil permitan impulsar, como indican Rex o Habermas, una identidad cívica lo suficientemente permeable para incorporar la diferencia respetando su integridad y potenciar el desarrollo de un modelo social que compagine la pluralidad en las formas de vida y una cultura política común.

\section{BIBLIOGRAFÍA}

AdelmAn, H. (ed.) (1995): Legitimate and Illegitimate Discrimination: New Issues in Immigration, Toronto, York Lanes Press.

Arango, J. (1994): «La cuestión migratoria en la Europa de fines del siglo XX», en NADAL, J. (ed.), El Mundo que viene, Madrid, Alianza, pp. 63-94.

${ }^{22}$ En este modelo, como señala Bauböck, las barreras culturales de la ciudadanía se mantienen - abiertas a los cambios en las afiliaciones y se promueve la admisión "superando la cultura nacional dominante». Se trata, por tanto, de una propuesta que no condiciona la inclusión a la previa afiliación cultural y política de los inmigrantes y que presupone que el acceso producirá fuertes transformaciones en las identidades colectivas tanto de los grupos mayoritarios como de las minorías (R. Bauböck, 1998).

${ }^{23}$ Véase A. M. López Sala, Inmigrantes y Estados: la respuesta política ante la cuestión migratoria, Barcelona, Anthropos, 2002 (en prensa). 
BauBöCK, Rainer (1994a): Transnational Citizenship. Membership and Rights in International Migration, Aldershot, UK, Edward Elgar.

- (1994b) (ed.): From Aliens to Citizens. Redefining the status of immigrants in Europe, Avebury, Aldershot.

Bauböck, R.; Heller, A., y Zolberg, A. (1996) (eds.): The Challenge of Diversity. Integration and Pluralism in Societies of Immigration, Aldershot, Avebury.

BAuböck, R., y Rundell, J. (1998) (eds.): Blurred Boundaries: Migration, Ethnicity, Citizenship, Aldershot, Ashgate.

BAUBÖCK, R. (2002): «How migration transforms citizenship: international, multinational and transnational perspectives», en Simposio Internacional Europa y el Mediterráneo. Políticas de Inmigración, Barcelona, Organizado por la Universidad Pompeu Fabra y el Instituto Catalá de la Mediterranía.

BRUBAKER, W. R. (1990): «Immigration, Citizenship and the Nation State in France and Germany: A Comparative Historical Analysis», en International Sociology, vol. 5, núm. 4, pp. 379-407.

Carens, J. H. (1995): «Immigration, Welfare and Justice», en Schwartz, W. F. (ed.), Justice in Immigration, Cambridge, Cambridge Studies in Philosophy and Law, Cambridge University Press, pp. 1-17.

Castles, S., y Miller, M. (1993): The Age of Migration. International Populations Movements in the Modern World, Londres, MacMillan.

CAstles, S., y Davidson, A. (2000): Citizenship and Migration. Globalization and the Politics of Belonging, Londres, MacMillan Press.

CASTLES, S. (2002): «Globalization and Immigration», en Simposio Internacional Europa y el Mediterráneo. Políticas de Inmigración, Barcelona, Organizado por la Universidad Pompeu Fabra y el Instituto Catalá de la Mediterranía.

Cornelius, W. A.; Martin, P. L., y Hollifiled, J. F. (1995a): Controlling Immigration: A Global Perspective, Stantford, Stantford University.

FAIST, T. (1996): «Immigration, Integration and the Welfare State: Germany and USA in a comparative perspective», en BAuböck, R.; Heller, A., y ZolberG, A. (eds.), The Challenge of Diversity: Integration and Pluralism in Societies of Immigration, Warwick, Avebury, pp. 227-259.

Flores D'arcais, P. (2002): Estado de alerta en Europa, El País, domingo 28 de abril de 2002.

FreEman, G. (1997): «Immigration as a Source of Political Discontent and Frustation in Western Democracies», en Studies in Comparative International Development, vol. 32, núm. 3, pp. 42-64.

García, S. (1994): «Ciudadanía en España», en García, S.; Giner, S., y Alabart, A. (eds.), Clase, poder y ciudadanía, Barcelona, Siglo XXI, pp. 223-245.

Gellner, E. (1988): Naciones y Nacionalismo, Madrid, Alianza Universidad.

Gimeno GonZÁlez, L. (2001): Actitudes ante la inmigración. Relación entre las investigaciones cualitativas y cuantitativas, Colección Opiniones y Actitudes, núm. 34, Madrid, CIS.

Hammar, T. (1985): European Immigration Policy: a Comparative Study, Nueva York, Cambridge University Press.

- (1990): Democracy and the Nation State: Aliens. Denizens and Citizens in a World of International Migration, Aldershot, Avebury.

Hollifield, J. (1992): Immigrants, Markets and States, Cambridge, Massachusses, Harvard University Press. 
- (1998): «Ideas, Institutions and Civil Society: On the Limits of Immigration Control in Liberal Democracies», Seminario sobre Inmigración y Estado, Center for Migration, Ethnicity and Citizenship, Nueva York, New School University.

- (2000): «The Politics of International Migration. How can we bring the State Back in», en BretTel, C., y Hollifield, J. (eds.), Migration Theory. Talking across disciplines, Londres, Routledge, pp. 137-185.

JACOBSON, D. (1996): Rights across Borders: Immigration and the Decline of Citizenship, Baltimore, John Hopkins University Press.

JOPPKE, C. (1999): Immigration and the Nation State: The United States, Germany and Great Britain, Oxford, Oxford University Press.

Koopmans, R., y Statham, P. (2000) (eds.): Challenging Immigration and Ethnic Relations Politics, Oxford, Oxford University Press.

Kymlicka, W. (1995): Multicultural Citizenship, Oxford, Clarendon Press.

López SALA, A. M. (2001): «La gestión política de la inmigración», en Colom, F. (ed.), El espejo, el crisol y el mosaico. Modelos políticos para el Multiculturalismo, Barcelona, Anthropos.

- (2002): Inmigrantes y Estados: la respuesta política ante la cuestión migratoria, Barcelona, Anthropos (en prensa).

Martiniello, M. (1995): Migration, Ethnicity and Ethno-National Identities in the European Union, Aldershot, Avebury.

RAMIREZ, F., y BoLl, J. (1987): «The political construction of mass schooling: European origins and worldwide institutionalization», en Sociology of Education, vol. 60, pp. 2-17.

Rawls, J. (1972): A Theory of Justice, Oxford, Clarendon Press.

ReX, J. (1996): Ethnic Minorities in the Modern Nation State, Londres, MacMillan.

Rubro, R. (2000): Immigration as a Democratic Challenge. Citizenship and Inclusion in Germany and the United States, Cambridge, Cambridge University Press.

SASSEN, S. (2001): ¿Perdiendo el control? La soberanía en la era de la globalización, Barcelona, Ediciones Bellaterra.

SCHNAPPER, D. (1992): L'Europe des immigrés. Essai sur les politiques d'immigration, París, F. Bourin.

Sciortino, G. (2000): «Towards a political sociology of entry policies: conceptual problems and theoretical proposals», en Journal of Ethnic and Racial Studies, vol. 26, núm. 2, pp. 213-228.

Simon, R., y LyNCH, J. (1999): «A Comparative Assessment of Public Opinion toward Immigrants and Immigration Policies», en International Migration Review, vol. 22, núm. 2, pp. 455-467.

SOYSAL, Y. (1994): Limits of Citizenship: Migrants and Postnational Membership in Europe, Chicago, Chicago University Press.

- (1996): «Changing Citizenship in Europe: Remarks on postnational Membership and The National State», en Cesaran,, D., y Fullbrook, M. (eds.), Citizenship, Nationality and Migration in Europe, Londres, Routledge, pp. 17-29.

- VAN AMERSFOORT, H. (1996): «Migration: the Limits of Government Control», en New Community, vol. 22, núm. 2, pp. 243-257.

VERTOVEC, S. (1999): «Minority associations, networks and public policies; re-assessing relationships», en Journal of Ethnic and Racial Studies, vol. 25, núm. 1, pp. 21-42.

WALzER, M. (1983): Spheres of Justice, Nueva York, Basic Books, traducción castellana, Esferas de justicia, México, FCE, 1993. 
Westin, C., y DACYL, J. (eds.): Governance of Cultural Diversity: Selected Aspects, Center for Research in International Migration and Ethnic Relations, Suecia, Universidad de Estocolmo.

Whitol de Wenden, C., y Hargreaves, A. (1993): «The Political Participation of Ethnic Minorities in Europe: A framework for analysis», en New Communitty, vol. 1, núm. 20 , pp. 1-8.

Young, I. (1989): «Polity and Group Difference: A Critique of the Ideal of Universal Citizenship», en Ethics, núm. 99, pp. 250-274.

ZAPATA, R. (2000): «Inmigración e innovación política», en Revista Migraciones, núm. 8, pp. 7-58.

Zolberg, A. (1981): «International Migrations in Political Perspective», en KrITZ, M.; KeEly, C., y Tomasi, S. (eds.), Global Trends in Migration: Theory and Research on International Populations Movements, Center for Migration Studies, Nueva York, Staten Island, pp. 3-27.

- (1989): «The Next Waves: Migration Theory for a Changing World», en International Migration Review, vol. 23, núm. 3, pp. 403-430.

- (1999): «Matters of State: Theorizing Immigration Policy», en HirschMan, C.; KasINITZ, P., y DE WIND, J. (eds.), The Handbook of International Migration. The American Experience, Londres, Russell-Sage, pp. 71-93. 